\title{
Taut String Model: Getting the Right Energy versus Getting the Energy the Right Way
}

\author{
Zach Caamaño-Withall, Petr Krysl \\ Department of Structural Engineering, University of California, San Diego, La Jolla, CA, USA \\ Email: pkrysl@ucsd.edu
}

Received 29 December 2015; accepted 26 February 2016; published 29 February 2016

Copyright (C) 2016 by authors and Scientific Research Publishing Inc.

This work is licensed under the Creative Commons Attribution International License (CC BY). http://creativecommons.org/licenses/by/4.0/

(c) (i) Open Access

\begin{abstract}
The initial boundary value problem of the transverse vibration of a taut string is a classic that can be found in many vibration and acoustics textbooks. It is often used as the basis for derivations of elementary numerical models, for instance finite element or finite difference schemes. The model of axial vibration of a prismatic elastic bar also serves in this capacity, often times side-by-side with the first model. The stored (potential) energy for these two models is derived in the literature in two distinct ways. We find the potential energy in the taut string model to be derived from a second-order expression of the change of the length of the string. This is very different in nature from the corresponding expression for the elastic bar, which is predictably based on the work of the internal forces. The two models are mathematically equivalent in that the equations of one can be obtained from the equations of the other by substitution of symbols such as the primary variable, the resisting force and the coefficient of the stiffness. The solutions also have equivalent meanings, such as propagation of waves and standing waves of free vibration. Consequently, the analogy between the two models can and should be exploited, which the present paper successfully undertakes. The potential energy of deformation of the string was attributed to the seminal work of Morse and Feshbach of 1953. This book was also the source of a misunderstanding as to the correct expression for the density of the energy of deformation. The present paper strives to settle this question.
\end{abstract}

\section{Keywords}

Taut String, Transverse Vibration, Potential Energy

\section{Introduction}

The study of transverse waves propagating in infinite or finite taut strings often contributes to the foundations in undergraduate curricula concerned with vibration and sound. The model frequently appears in graduate and 
undergraduate textbooks [1]-[4]. It is commonly used as basis for derivations of elementary numerical models for initial boundary value problems, for instance finite element or finite difference schemes.

The model of axial vibration of a prismatic elastic bar also fulfills these functions, oftentimes side-by-side with the first model. The two models are mathematically equivalent in that the equations of one can be obtained from the equations of the other by substitution of symbols such as the primary variable and the resisting force. The analogy between the two models can and should be exploited to reinforce the reader' understanding of the workings of the models and of the solution processes.

Contrary to expectation, the two models despite being equivalent in all other respects are at variance in the derivations of the stored (strain, potential) energy. The taut string model potential energy is derived from a second-order expression of the length of the string, whereas the potential energy stored in the axially deformed bar is derived using an argument based on the work of the resisting forces. The second-order-effect explanation of the source of potential energy stored in the taut string only serves to confuse, since the change in length of the string plays no role in the rest of the model. The present work shows that such a device is unnecessary and can be replaced with a derivation that perfectly parallels the model of axial deformation of an elastic bar. The correct expression for the potential energy is recovered and we claim that it is derived in a way that is of most utility in applications in vibrations and acoustics.

In Section 2, we introduce the notion that boundary-value problems described by analogous equations can be derived following analogous steps. Namely in our investigation we are interested in deriving the expression for the potential energy of deformation. The two analogous models are the model of axial deformation of an elastic bar and the taut-string model.

In Section 3, we derive the potential energy of deformation stored in an axially deformed elastic bar by applying a thought loading process and tracking the work of the forces acting on the differential element of the bar on the displacement increments associated with the progression through the loading process. The expressions that we obtain in this way are then re-interpreted in Section 2 for the string boundary value problem.

In Section 5, we review the literature going back to the main sources which include the famous Rayleigh treatise The Theory of Sound, but also touch upon a fairly recent string of papers that address various aspects of the derivations of the potential energy of deformation of the taut string. We critically examine the evolution of the expression derived for the potential energy of deformation in the influential literature of the last century and we correlate our approach with the established arguments. We show that even though the end product is, as could be expected, the same as that derived by Rayleigh, the process, especially in textbooks, is elucidated in the alternative way we propose.

\section{Bar and String: Analogous Models}

The derivations in this work are produced for a static model of deformation. That is not a limitation as the potential energy of deformation for the two discussed present models does not depend on the velocities or accelerations by definition. Consequently the present derivations are directly applicable to models of transient or free vibration, both for the axial bar treated in this section and for the model of a taut string treated in the next section.

The model of the axial deformation of the linearly elastic bar is described by the following statements:

1) The bar is prismatic of cross-sectional area $A$ and straight of length $L$. The coordinate $0 \leq x \leq L$ is the independent variable measured along the axis of the bar.

2) The cross-sections of the bar remain planar and the points within the cross-sections move by displacement $u(x)$ only along the axis of the bar. The magnitude of the displacements is negligible compared to the length of the bar, $|u(x)| \ll L$, and we also assume the strains to be small, $|\partial u(x) / \partial x| \ll 1$.

$3)$ The material is linearly elastic, described by the modulus of elasticity $E$.

4) The elastic resisting force is expressed as

$$
F(x)=E A \frac{\partial u(x)}{\partial x} .
$$

5) The bar is loaded by distributed axial force $b(x)$ in units of force/length.

6) The equilibrium of a differential element of the bar is written as 


$$
\frac{\partial F(x)}{\partial x}+b(x)=0
$$

7) The boundary conditions are expressed either in terms of prescribed displacement or in terms of applied force. Denoting the cross-section where the displacement is prescribed as $S_{1}$ and the cross-section where the force is prescribed as $S_{2}$ we can write

$$
u \mid S_{1}=\text { given value }
$$

and

$$
F \mid S_{2}=\text { given value. }
$$

The model will be referred to in the following as BAR.

The model of the planar transverse deformation of the taut string is the model we wish to investigate in this paper with respect to the derivation of the potential energy of deformation. The model is described by the following statements:

1) The string of length $L$ is initially straight. The coordinate $0 \leq x \leq L$ is the independent variable measured along the axis of the string.

2) The points along the centerline of the string move by displacement $w(x)$ only transversely to the axis of the string and we assume all points of the string move in a single plane. The magnitude of the displacements is negligible compared to the length of the string, $|w(x)| \ll L$, and we also assume the magnitude of the slope of the string to be small, $|\partial w(x) / \partial x| \ll 1$.

3 ) The string is prestressed by a uniform and unchanging force $P$.

4) The resisting (transverse) force is expressed as

$$
S(x)=P \frac{\partial w(x)}{\partial x} .
$$

5) The string is loaded by distributed transverse force $q(x)$ in units of force/length.

6) The equilibrium of a differential element of the bar is written as

$$
\frac{\partial S(x)}{\partial x}+q(x)=0 .
$$

7) The boundary conditions are expressed either in terms of prescribed displacement or in terms of applied force. Denoting the cross-section where the displacement is prescribed as $S_{1}$ and the cross-section where the force is prescribed as $S_{2}$ we can write

$$
w \mid S_{1}=\text { given value }
$$

and

$$
S \mid S_{2}=\text { given value. }
$$

The model will be referred to in the following as STRING.

We see that the two boundary value problems, BAR and STRING, are described by the same kinds of equations. We can get one boundary value problem from the other by the substitution

$$
u \Leftrightarrow w, F \Leftrightarrow S, b \Leftrightarrow q, E A \Leftrightarrow P .
$$

Therefore, we expect to be able to derive the potential energy of deformation for the BAR model and then obtain the potential energy of deformation for the STRING model by the symbol substitution (9). Even more useful is the ability to repeat the derivation for the STRING model using the substitution (9) applied to the derivation performed for the BAR model and thereby extract a physical understanding of the quantities involved and the meaning of the actions in which they participate.

\section{Energy of Deformation for the BAR Model}

Elementary vibration textbooks typically include a derivation of the potential energy that is developed like this: 
Consider a linear spring (Figure 1). The thought process of loading describes the elongation of the spring as $y(\theta)=\theta y$, and the resisting force is therefore $F(\theta)=k y(\theta)=\theta k y$, where $y$ is the target elongation of the spring, not necessarily the current elongation. As the loading parameter is increased from $\theta$ to $\theta+d \theta$, the force $F(\theta)=\theta$ ky works on the increment of displacement $y(\theta+d \theta)-y(\theta)=d \theta y$ to give the work increment $\theta k y \times y \times d \theta$. Upon integration with respect to $\theta$ we obtain the familiar result $W=(1 / 2) k y^{2}=(1 / 2) F y$. Figure 1 clearly shows that only half of the work of the force $F$ on the elongation of the spring $y$ is stored as energy of deformation: the reason is that the restoring force $F$ is proportional to $y$, and the slanted line in the elongationforce diagram must be followed during loading or unloading.

We believe that a consistent elementary argument should be patterned on the above reasoning. We shall now proceed to demonstrate the derivation of the potential energy of deformation on the BAR model.

We shall assume that the potential energy of deformation is sought for a given displacement function $u(x)$. We will imagine a process where the final shape is achieved by progressively increasing the displacement amplitude from zero to the final value. This application will be described using a load parameter $\theta$, whose value $\theta=0$ corresponds to the undeformed bar, and $\theta=1$ corresponds to the target value of the displacement.

For simplicity we shall assume that the dependence on the load parameter is linear, so that we have for the displacement

$$
u(x, \theta)=\theta u(x)
$$

and the body load is also ramped up linearly

$$
b(x, \theta)=\theta b(x) .
$$

Importantly, substituting (10) into the definition of the restoring force $F$ results in

$$
F(x, \theta)=\theta F(x) .
$$

Consequently, during the process of imposing the target deformation the equilibrium of any differential element of the bar is maintained. Figure 2 shows the displacements and forces on a generic infinitesimal element of the bar at the value of the loading parameter $\theta$.

Consider now an increase of the loading parameter from $\theta$ to $\theta+d \theta$, which results in an increase of the displacement in the left-hand-side cross-section to

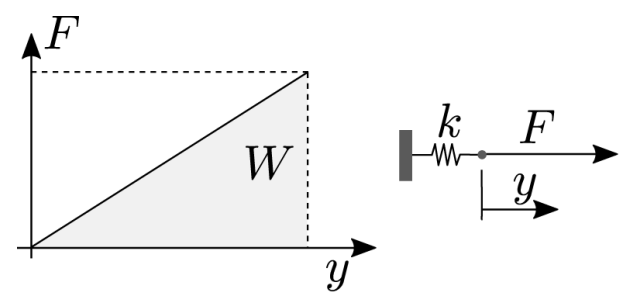

Figure 1. Linear spring: Energy of deformation.

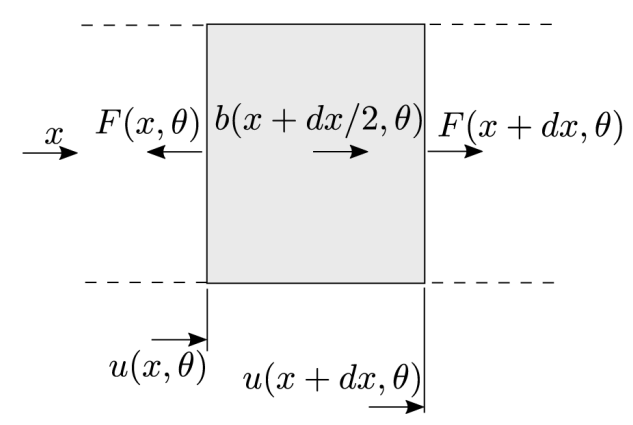

Figure 2. Schematic of a differential element of the bar, forces and displacements indicated. 


$$
u(x, \theta+d \theta)=u(x, \theta)+\frac{\partial u(x, \theta)}{\partial \theta} d \theta .
$$

Using Equation (10) allows us to rewrite (13)

$$
u(x, \theta+d \theta)=(\theta+d \theta) u(x)
$$

so that the increment of the displacement from $\theta$ to $\theta+d \theta$ in the left-hand-side cross-section is

$$
u(x, \theta+d \theta)-u(x, \theta)=u(x) d \theta .
$$

Similarly in the right-hand-side cross-section the displacement increases to

$$
u(x+d x, \theta+d \theta)=u(x+d x, \theta)+\frac{\partial u(x+d x, \theta)}{\partial \theta} d \theta
$$

which leads analogously to the increment of the displacement in the right-hand-side cross-section

$$
u(x+d x, \theta+d \theta)-u(x+d x, \theta)=u(x+d x) d \theta
$$

Using the Taylor expansion in $x$ for the displacement we can re-write (17)

$$
u(x+d x, \theta+d \theta)-u(x+d x, \theta)=\left[u(x)+\frac{\partial u(x)}{\partial x} d x\right] d \theta
$$

Let us now look at the left-hand-side cross-section: The force acting here works during the incremental change of the displacement from $\theta$ to $\theta+d \theta$

$$
-F(x, \theta)[u(x, \theta+d \theta)-u(x, \theta)]
$$

where the negative sign indicates that the force in the left-hand-side cross-section points against the $x$ axis. Substituting (15) results in

$$
-F(x, \theta)[u(x, \theta+d \theta)-u(x, \theta)]=-F(x, \theta) u(x) d \theta
$$

Analogously, in the right-hand-side cross-section the restoring force $F(x+d x, \theta)$ works on the incremental displacement as

$$
+F(x+d x, \theta)[u(x+d x, \theta+d \theta)-u(x+d x, \theta)]
$$

Substituting (18), we obtain

$$
+F(x+d x, \theta)\left[u(x)+\frac{\partial u(x)}{\partial x} d x\right] d \theta
$$

where the force can also be expanded in a Taylor series

$$
F(x+d x, \theta)=F(x, \theta)+\frac{\partial F(x, \theta)}{\partial x} d x
$$

to yield for (22)

$$
+\left[F(x, \theta)+\frac{\partial F(x, \theta)}{\partial x} d x\right]\left[u(x)+\frac{\partial u(x)}{\partial x} d x\right] d \theta
$$

The work performed on the differential element of Figure 2 by the restoring forces is the sum of the contributions (20) and (24)

$$
+\left[F(x, \theta)+\frac{\partial F(x, \theta)}{\partial x} d x\right]\left[u(x)+\frac{\partial u(x)}{\partial x} d x\right] d \theta-F(x, \theta) u(x) d \theta
$$


which can be approximated by canceling and omitting the second-order term in the differential $d x$ as

$$
\frac{\partial F(x, \theta)}{\partial x} u(x) d x d \theta+F(x, \theta) \frac{\partial u(x)}{\partial x} d x d \theta
$$

Next we will consider the distributed body load. The body load works on the increment of the displacement of the differential element from $\theta$ to $\theta+d \theta$ as

$$
b(x, \theta)[u(x, \theta+d \theta)-u(x, \theta)] d x=b(x, \theta) u(x) d \theta d x
$$

A more complicated approximation formula could have been used, such as the midpoint rule or the trapezoidal rule. However, the result (27) would still stand as we would have to neglect all terms with higher powers of the differentials than linear.

The work of all the forces acting on the differential element of the bar between $\theta$ and $\theta+d \theta$ is the sum of (26) and (27)

$$
W(x) d x d \theta=\frac{\partial F(x, \theta)}{\partial x} u(x) d x d \theta+F(x, \theta) \frac{\partial u(x)}{\partial x} d x d \theta+b(x, \theta) u(x) d \theta d x
$$

which may be regrouped as

$$
W(x) d x d \theta=\left[\frac{\partial F(x, \theta)}{\partial x}+b(x, \theta)\right] u(x) d x d \theta+F(x, \theta) \frac{\partial u(x)}{\partial x} d x d \theta .
$$

Using (11) and (12), we see that the bracket will vanish

$$
\frac{\partial F(x, \theta)}{\partial x}+b(x, \theta)=\theta\left[\frac{\partial F(x)}{\partial x}+b(x)\right]=0
$$

because the balance Equation (2) is satisfied. Consequently, the work of all the forces acting on the differential element of the bar between $\theta$ and $\theta+d \theta$ is seen to be

$$
W(x) d x d \theta=F(x, \theta) \frac{\partial u(x)}{\partial x} d x d \theta=\theta F(x) \frac{\partial u(x)}{\partial x} d x d \theta
$$

where we have used (12) again. The work performed on the differential element over the entire loading process is obtained by integration with respect to $\theta$

$$
W(x) d x=\int_{0}^{1} \theta F(x) \frac{\partial u(x)}{\partial x} d x \mathrm{~d} \theta=\frac{1}{2} F(x) \frac{\partial u(x)}{\partial x} d x .
$$

This work is stored in the differential element of the bar as energy of deformation. The density of the energy of deformation is consequently the expression

$$
W(x)=\frac{1}{2} F(x) \frac{\partial u(x)}{\partial x} .
$$

Substituting the definition of the axial force (1) reduces the above to the familiar formula

$$
W(x)=\frac{1}{2} E A\left[\frac{\partial u(x)}{\partial x}\right]^{2}
$$

Note that the potential energy of deformation of the entire bar can be obtained by integration over the length $0 \leq x \leq L$.

\section{Energy of Deformation for the STRING Model}

As advertised, the deformation energy for the STRING model can be written immediately from (34) by 
exchange of the symbols as defined in (9)

$$
W(x)=\frac{1}{2} P\left[\frac{\partial w(x)}{\partial x}\right]^{2} .
$$

More importantly, we can now unravel the physical meaning that leads from the equilibrium of the differential element as shown in Figure 3 to the expression (9) for the energy of deformation of the string.

Figure 3 shows the deflections and forces acting on a differential element of the taut string at the load parameter $\theta$ of the imagined loading process. As for the BAR model, we will now form a similar force diagram for the load parameter value $\theta+d \theta$, and the increment of displacement of the left-hand-side point will be analogous to (15)

$$
w(x, \theta+d \theta)-w(x, \theta)=w(x) d \theta
$$

and the increment of displacement of the right-hand-side point will be

$$
w(x+d x, \theta+d \theta)-w(x+d x, \theta)=\left[w(x)+\frac{\partial w(x)}{\partial x} d x\right] d \theta
$$

The transverse force $S$ works on the increments of displacement at the two endpoints of the element so that using the steps outlined above Equation (29) we arrive at

$$
W(x) d x d \theta=\left[\frac{\partial S(x, \theta)}{\partial x}+q(x, \theta)\right] w(x) d x d \theta+S(x, \theta) \frac{\partial w(x)}{\partial x} d x d \theta .
$$

Applying the balance Equation (29) results in

$$
W(x) d x d \theta=S(x, \theta) \frac{\partial w(x)}{\partial x} d x d \theta,
$$

in complete parallel to Equation (31). We can see that the restoring force $S(x)$ works on the strain-like quantity $\partial w(x) / \partial x$ which for the STRING model is the slope of the deflection curve. The restoring force is related to the strain-like slope by the constitutive Equation (5), and so the density can be written immediately after integration with respect to $\theta$ as

$$
W(x)=\frac{1}{2} P\left[\frac{\partial w(x)}{\partial x}\right]^{2} .
$$

The energy of deformation in the entire string is of course obtained by integration along the length

$$
W=\int_{0}^{L} \frac{1}{2} P\left[\frac{\partial w(x)}{\partial x}\right]^{2} \mathrm{~d} x
$$

\section{Discussion}

Morse and Feshbach [5] are often cited where the model of the transverse oscillation of the taut string model is

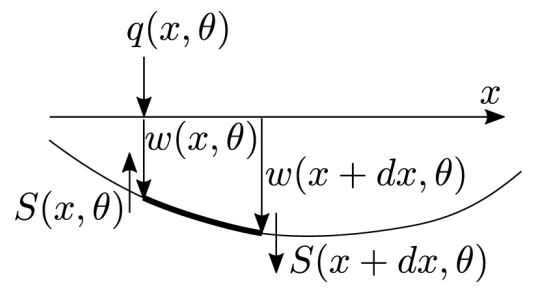

Figure 3. Schematic of a differential element of the string, forces and displacements indicated. 
concerned. The book contains two derivations of the density of the energy of deformation. We have been able to follow these two arguments to their apparent source of [6] (original publication from 1925 cited in [5]; the Dover edition presents this derivation on pages 60 - 61). Both of these arguments are reproduced in [7] (which first appeared in 1936) and then also later in [5].

In the first approach to the calculation of the potential energy of deformation Morse and Feshbach [5] state in Chapter 2, page 126 (we use our symbols):

The applied force on an element $d x$ of the string in order to have reached the intermediate shape $\theta w$ is $-P \theta\left(\partial^{2} w / \partial x^{2}\right) d x$, and the work done by this force to move this element from $\theta w$ to $(\theta+d \theta) w$ is $-P w\left(\partial^{2} w / \partial x^{2}\right) d x \theta d \theta$.

This leads to the density of deformation energy

$$
W(x)=-\frac{1}{2} P w(x) \frac{\partial^{2} w(x)}{\partial x^{2}} .
$$

The deformation energy of the entire string is consequently obtained by integration as

$$
W=\int_{0}^{L}-\frac{1}{2} P w\left(\partial^{2} w / \partial x^{2}\right) \mathrm{d} x
$$

Then in order to arrive at a symmetric expression (as in our Equation (41)), integration by parts is applied, which in addition to the integral of the energy density (41) also includes a boundary term (i.e. product of slope and deflection at the ends of the string)

$$
W=\int_{0}^{L} \frac{1}{2} P\left[\frac{\partial w(x)}{\partial x}\right]^{2} \mathrm{~d} x-\left[P w(x) \frac{\partial w(x)}{\partial x}\right]_{0}^{L}
$$

The argument leading to (42) will be in the following referred to as CURV.

Expression (44) is undoubtedly wrong. The boundary term vanishes for homogeneous boundary conditions, but does not in general vanish for inhomogeneous boundary conditions and thus the energy of deformation becomes dependent on the rigid body motion of the string. For arbitrary values of $\partial w(0) / \partial x \neq \partial w(L) / \partial x$ and rigid body translation added to the deflection $w(x) \rightarrow w(x)+A$, the energy changes by the nonzero amount

$$
-P A\left[\frac{\partial w(x)}{\partial x}\right]_{0}^{L} \neq 0 .
$$

Also, we can highlight the problem directly from (42) by adding rigid body translation $A$ to the deflection curve $w(x)$ which changes the energy density to

$$
W(w(x)+A)=-\frac{1}{2} P[w(x)+A] \frac{\partial^{2} w(x)}{\partial x^{2}}=W(w(x))-\frac{1}{2} P A \frac{\partial^{2} w(x)}{\partial x^{2}} .
$$

Equation (42) cannot be the correct energy of deformation density as it depends on rigid body motion. Furthermore, expression (44) mixes together potential energy of two different kinds: the potential energy of deformation and the potential energy of the applied loads (or work). Equation (44) is in substantial disagreement with the customary meaning of variational methods, such as the principle of total potential energy [8].

As the authors of [5] were slightly uncomfortable with the apparently ambiguous expression for the potential energy of deformation of the string (44), they also presented a second expression for the energy on page 127. Here, the argument (referred to by the abbreviation ELONG in what follows) was made that the differential element of the string becomes longer during deformation and the (unchanging) prestressing force works on this elongation. The differential element of the string that was originally of length $d x$ will assume upon deformation the length (Figure 4).

$$
d s=d x \sqrt{1+\left(\frac{\partial w(x)}{\partial x}\right)^{2}} \approx d x\left[1+\frac{1}{2}\left(\frac{\partial w(x)}{\partial x}\right)^{2}\right]
$$




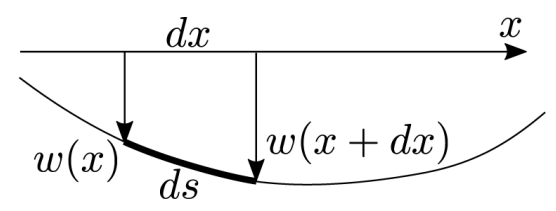

Figure 4. Differential element of the string becoming longer as the string deforms.

It is therefore concluded in [5] that the work of the prestressing force $P$ on the elongation $(d s-d x)$ represents the density of the energy of deformation

$$
W(x)=P \frac{1}{2}\left(\frac{\partial w(x)}{\partial x}\right)^{2} .
$$

Argument ELONG was already advanced by Rayleigh [9] (appeared in 1894), which was possibly the source for the treatment in Lamb [6]. Established monographs [10]-[15] and many recent textbooks repeat the argument ELONG (almost) verbatim, for instance [1]-[4].

The matter of the energy of deformation of the taut string has also been subject to some critical examinations over the years. Matthews [16] derived the CURV equation, and also struggled with the ensuing non-uniqueness; the author did not realize that the expression for the density was wrong. Burko [17] derived the density of energy of deformation to arrive at the two versions of the energy density of [5], and then concluded that both expressions CURV and ELONG for the energy density were correct and that the ambiguity of the energy definitions did not matter. Butikov [18] criticized these conclusions, but also stated that the potential energy of the string resulted from the elastic stretching (ELONG argument). Rowland [19] concluded that Morse and Feshbach were correct, stating that the potential energy of deformation defined using the curvature was non-unique, and on that basis rejected the energy density defined using curvature (the CURV argument); the more fundamental problem with rigid body translation was not realized in [19] though.

Formula (48) (obtained using the argument ELONG) is identical to our (44). The result is undoubtedly correct, but that does not mean that the derivations are mutually replaceable. We believe an elementary treatment should follow the pattern outlined in Section 3. The problems with the ELONG argument are the following:

1) The prestressing force $P$ is not the restoring force. By assumption $P$ is an input (property). It does not react to the elongation of the string (which varies from point to point). In order for researchers to appreciate this distinction they need to be aware of the (second-order, nonlinear) theory of the response of structures under prestressing (initial) forces/stresses.

2) Elongation is not needed anywhere in the model and is solely enlisted in the calculation of the energy of deformation, where it makes its appearance almost as an invocation of deus ex machina.

3) The fact that the density of the energy of deformation is the entire product of (force $\times$ elongation) instead of one half of this product is a potential source of confusion to readers of elementary treatments. The fraction $1 / 2$ that appears in (48) is part of the relative elongation, it is not employed to take one half of the product (force $\times$ elongation) (i.e. the area of the triangle underneath the force-displacement triangle in Figure 1).

\section{Conclusion}

The derivation of the potential energy of deformation for the transverse deflection of a taut string was carried out in complete analogy with the equivalent model of the axial deformation of a linearly elastic prismatic bar. The resulting expression is the same as that which appears in many textbooks and monographs since the days of Rayleigh and his famous treatment in the Theory of Sound. However, the path followed is different. We believe that the consistent derivation of the potential energy of deformation from the equilibrium of a differential element provides the clarity and insight from which users of elementary treatments of vibrations and sound can only benefit.

\section{References}

[1] Rao, S. (2007) Vibration of Continuous Systems. Wiley, Hoboken. 
[2] Virgin, L. (2007) Vibration of Axially-Loaded Structures. Cambridge University Press, Cambridge. http://dx.doi.org/10.1017/CBO9780511619236

[3] Bishop, R. and Johnson, D. (2011) The Mechanics of Vibration. Cambridge University Press, Cambridge.

[4] Gatti, P. (2014) Applied Structural and Mechanical Vibrations: Theory and Methods. 2nd Edition, Taylor \& Francis, London. http://dx.doi.org/10.1201/b16697

[5] Morse, P. and Feshbach, H. (1953) Methods of Theoretical Physics, no. v. 1 in International Series in Pure and Applied Physics. McGraw-Hill, Boston.

[6] Lamb, H. (2004) The Dynamical Theory of Sound. Dover Phoenix Editions, Dover Publications, Mineola.

[7] Morse, P. (1986) Vibration and Sound. American Institute of Physics for the Acoustical Society of America, College Park.

[8] Washizu, K. (1974) Variational Methods in Elasticity and Plasticity. 2nd Edition, Pergamon Press Ltd., Oxford.

[9] Rayleigh, J. and Lindsay, R. (1945) The Theory of Sound. Dover Publications, Inc., Dover.

[10] Graff, K. (2012) Wave Motion in Elastic Solids, Dover Books on Physics. Dover Publications, Mineola.

[11] Elmore, W. and Heald, M. (2012) Physics of Waves, Dover Books on Physics. Dover Publications, Mineola.

[12] Morse, P. and Ingard, K. (1968) Theoretical Acoustics, International Series in Pure and Applied Physics. Princeton University Press, Princeton.

[13] Giaquinta, M. and Hildebrandt, S. (1996) Calculus of Variations I: The Lagrangian Formalism. Springer, Berlin.

[14] Selvadurai, A. (2000) Partial Differential Equations in Mechanics 1. Springer Berlin Heidelberg, Berlin.

[15] Arnold, V. (2004) Lectures on Partial Differential Equations. Universitext (1979), Springer, Berlin. http://dx.doi.org/10.1007/978-3-662-05441-3

[16] Mathews, W.N. (1985) Energy in a One-Dimensional Small Amplitude Mechanical Wave. American Journal of Physics, 53, 974-978. http://dx.doi.org/10.1119/1.14405

[17] Burko, L.M. (2010) Energy in One-Dimensional Linear Waves in a String. European Journal of Physics, 31, L71. http://dx.doi.org/10.1088/0143-0807/31/5/L01

[18] Butikov, E.I. (2011) Comment on “Energy in One-Dimensional Linear Waves in a String”. European Journal of Physics, 32, L35. http://dx.doi.org/10.1088/0143-0807/32/6/L01

[19] Rowland, D.R. (2013) Small Amplitude Transverse Waves on Taut Strings: Exploring the Significant Effects of Longitudinal Motion on Wave Energy Location and Propagation. European Journal of Physics, 34, 225-245. http://dx.doi.org/10.1088/0143-0807/34/2/225 